TRANSACTIONS OF THE

AMERICAN MATHEMATICAL SOCIETY

Volume 350, Number 3, March 1998, Pages 935-946

S $0002-9947(98) 01784-\mathrm{X}$

\title{
SOBOLEV ESTIMATES FOR OPERATORS GIVEN BY AVERAGES OVER CONES
}

\author{
SCIPIO CUCCAGNA
}

\begin{abstract}
We prove a result related to work by A. Greenleaf and G. Uhlmann concerning Sobolev estimates for operators given by averages over cones. This is done using the almost orthogonality lemma of Cotlar and Stein, and the van der Corput lemma on oscillatory integrals.
\end{abstract}

\section{INTRODUCTION}

This paper is inspired by [GU1, Theorem 3.23]. To state the result we need to introduce some notions, following [GU1]. Let $(M, g)$ be an $n$ dimensional riemannian manifold. We consider a $C^{\infty}$ map $\gamma^{0}: M \times S^{m-1} \times \mathbb{R} \rightarrow S(T M)$, with $S(T M)$ the unitary tangent bundle on $M$, such that for any $x \in \mathbb{R}^{n}$ the map $S^{m-1} \ni$ $\omega \rightarrow \gamma^{0}(x, \omega, 0) \in S\left(T_{x} M\right)$ is an embedding with image $\gamma_{x} \subset S\left(T_{x} M\right)$. We define $\gamma: M \times S^{m-1} \times \mathbb{R} \rightarrow M$ by $\gamma(x, \omega, r)=\exp _{x}\left(r \gamma^{0}(x, \omega, r)\right)$. Let $\Gamma=\{(x, \gamma(x, \omega, r)\}$ as the variables $(x, \omega, r)$ vary in the support of the distribution $K(\cdots)$ defined below. Consider now $K(x, \omega, r) \in \mathcal{D}^{\prime}\left(M \times S^{m-1} \times \mathbb{R}\right)$, a distribution with very small support whith the following properties.

First of all we fix a number $\mu$, with $0<\mu \leq m$. Then $K(x, \omega, r)$ is a smooth function for $r \neq 0$ satisfying the inequalities

$$
\left|\partial_{(x, \omega)}^{\alpha} \partial_{r}^{l} K(x, \omega, r)\right| \leq c_{\alpha, l} r^{-\mu-l}
$$

Moreover, if $\mu=m$, for any pair of small positive numbers $0<\epsilon<\eta$,

$$
\left|\int_{\epsilon<|r|<\eta} \partial_{(x, \omega)}^{\alpha} K(x, \omega, r) d r\right| \leq c_{\alpha},
$$

with the $c_{\alpha}$ independent of $\epsilon, \eta$. For $f \in C_{0}^{\infty}(M)$ we define

$$
\int_{S^{m-1} \times \mathbb{R}} K(x, \omega, r) f(\gamma(x, \omega, r)) r^{m-1} d \omega d r .
$$

Operators of this type are related to the operators of [GU1, (3.15)]. Operators of the form (0.2) appear naturally in the theory of x-ray transforms. If $\mathcal{M}$ is the manifold of geodesics of $(M, g)$ and if $\mathcal{C} \subseteq \mathcal{M}$ is an $n$ dimensional subspace, one can define for any $f \in C_{0}^{\infty}(M)$ the function $\mathcal{R}_{\mathcal{C}} f(p)$ which associates to each $p \in \mathcal{C}$ the average of $f(\cdot)$ along $p$. In important cases the operator $\mathcal{R}_{\mathcal{C}}^{t} \mathcal{R}_{\mathcal{C}}$ is of the form (0.2); see [GU1], [G]. Estimates on $\mathcal{R}_{\mathcal{C}}$ can be deduced from estimates on operators of the form (0.2).

Received by the editors September 30, 1994 and, in revised form, April 4, 1996.

1991 Mathematics Subject Classification. Primary 42B20, 26A33; Secondary 53C65.

Key words and phrases. Distributions, pseudodifferential operator, oscillatory integral, stationary phase, interpolation theorems for operators, conormal bundle. 
The most significant estimates depend on the geometry of $\mathcal{C}$, whose properties are reflected on $\Gamma$. We now restrict our attention to the case when $(M, g)$ is equal to $\mathbb{R}^{n}$ with the standard metric: the results of the type contained in this paper as well as [GU1, Theorem 3.23] are of local nature and can be easily transposed from this special case to more general riemannian manifolds.

Theorem 1. Assume $m>\mu$. If for any $x \in \mathbb{R}^{n}$ for any normal direction to $\gamma_{x}$ in $S^{n-1}$ the second fundamental form has rank at least $k$, for a fixed $k$ with $1 \leq k \leq m-1$, then for any $s \in \mathbb{R}$ the operator (0.2) extends to a bounded operator $T: L_{s}^{2}\left(\mathbb{R}^{n}\right) \rightarrow L_{s+s_{0}}^{2}\left(\mathbb{R}^{n}\right)$ if $s_{0} \leq \max (k / 2, m-\mu)$ when $m-\mu \neq k / 2$ and $s_{0} \leq k / 2-\delta$ for any $\delta>0$ when $m-\mu=k / 2$.

Theorem 2. Assume $\mu=m$. Suppose the $\gamma_{x}$ satisfy the same hypotheses as in Theorem 1. Then for any $p$ with $1<p<\infty$ the operator (0.2) extends to a bounded operator $T: L_{s}^{p}\left(\mathbb{R}^{n}\right) \rightarrow L_{s}^{p}\left(\mathbb{R}^{n}\right)$.

Theorem 3. Under the hypotheses of Theorem 2, if $m-k / 2<\mu$, then for any $s \in \mathbb{R}$ and for any $p$ with $|1 / p-1 / 2|<\frac{1}{2} \frac{k / 2-m+\mu}{k / 2+m-\mu}$ the operator (0.2) extends to a bounded operator $T: L_{s}^{p}\left(\mathbb{R}^{n}\right) \rightarrow L_{s-\mu+m}^{p}\left(\mathbb{R}^{n}\right)$.

The previous theorems for the case $k=m-1$ were proved in [G], [GU1] and [GU2]. The constant $\delta>0$ in Theorem 1 is necessary; see [GU2, pp. 435-6]. Our methods are quite different from those of these papers, and we mainly use some ideas from $[\mathrm{PS}]$ and $[\mathrm{SS}]$.

\section{Proof of Theorem 1. Preliminaries}

The Schwartz kernel of operator (0.2) is of the form

$$
K_{T}(x, y)=\int e^{i\langle\xi, \gamma(x, \omega, r)-y\rangle} a(x, y, \omega, r, \xi) r^{m-1} d \xi d \omega d r
$$

if $\mu<m$, or

$$
K_{T}(x, y)=\int e^{i\langle\xi, \gamma(x, \omega, r)-y\rangle+r \rho} a(x, y, \omega, r, \rho, \xi) d \xi d \omega d r d \rho
$$

if $\mu=m$. Here $a(x, y, \omega, r, \xi)=0$ and $a(x, y, \omega, r, \rho, \xi)=0$, if $(x, y, \omega, r)$ does not belong to some small compact set; also

$$
\left|\partial_{(x, y, \omega)}^{\alpha} \partial_{r}^{l} \partial_{\xi}^{\beta} a(x, y, \omega, r, \xi)\right| \leq c_{\alpha, l, \beta} r^{-\mu-l}(1+|\xi|)^{B-|\beta|}
$$

and

$$
\left|\partial_{(x, y, \omega, r)}^{\alpha} \partial_{\rho}^{l} \partial_{\xi}^{\beta} a(x, y, \omega, r, \rho, \xi)\right| \leq c_{\alpha, l, \beta}(1+|\rho|)^{-l}(1+|\xi|)^{B-|\beta|}
$$

with $B=0$.

Lemma 1.1. Let $T$ have kernel (1.1.a) resp. (1.1.b) with $B$ arbitrary in (1.2.a) resp. (1.2.b). Let $P$ be a pseudodifferential operator. Then PT and TP have, up to a smoothing operator, kernel of the form (1.1.a) resp. (1.1.b) with a(.) replaced by $a_{1}($.$) with$

$$
\left|\partial_{(x, y, \omega)}^{\alpha} \partial_{r}^{l} \partial_{\xi}^{\beta} a_{1}(x, y, \omega, r, \xi)\right| \leq c_{\alpha, l, \beta} r^{-\mu-l}(1+|\xi|)^{B+\operatorname{ord} P-|\beta|}
$$

resp.

$$
\left|\partial_{(x, y, \omega, r)}^{\alpha} \partial_{\rho}^{l} \partial_{\xi}^{\beta} a_{1}(x, y, \omega, r, \rho, \xi)\right| \leq c_{\alpha, l, \beta}(1+|\rho|)^{-l}(1+|\xi|)^{B+o r d} P-|\beta|
$$


Here each constant in (1.3.a) resp. (1.3.b) depends on finitely many constants in (1.2.a) resp. (1.2.b). Moreover, if $(\omega, r)$ does not belong to the set it has to belong to for $a(\cdots \omega, r, \cdots) \not \equiv 0$, then $a_{1}(\cdots, \omega, r, \cdots) \equiv 0$.

Proof. It is enough to consider the case when $a(x, y, \omega, r, \xi)$ resp. $a(x, y, \omega, r, \rho, \xi)$ has compact support. We consider $P_{x} K_{T}(x, y)$ and $Q_{y} K_{T}(x, y)$, with $Q$ the adjoint of $P$. Then

$$
P_{x} K_{T}(x, y)=\int e^{i\langle\xi, \gamma(x, \omega, r)-y\rangle} b(x, y, \omega, r, \xi) r^{m-1} d \xi d \omega d r
$$

resp.

$$
P_{x} K_{T}(x, y)=\int e^{i\langle\xi, \gamma(x, \omega, r)-y\rangle+\rho r} b(x, y, \omega, r, \rho, \xi) d \xi d \omega d r d \rho
$$

with

$$
b(x, y, \omega, r, \xi)=e^{-i\langle\xi, \gamma(x, \omega, r)\rangle} P\left\{e^{i\langle\xi, \gamma(., \omega, r)\rangle} a(., y, \omega, r, \xi)\right\}(x)
$$

resp.

$$
b(x, y, \omega, r, \rho, \xi)=e^{-i\langle\xi, \gamma(x, \omega, r)\rangle} P\left\{e^{i\langle\xi, \gamma(., \omega, r)\rangle} a(., y, \omega, r, \rho, \xi)\right\}(x) .
$$

More explicitly, our $b(x, y, \omega, r, \xi)$ resp. $b(x, y, \omega, r, \rho, \xi)$ is equal to

$$
e^{-i\langle\xi, \gamma(x, \omega, r)\rangle} \int e^{i[\langle\theta, x-z\rangle+\langle\xi, \gamma(z, \omega, r)\rangle]} p(x, z, \theta) a(z, y, \omega, r, \xi) d z d \theta
$$

resp.

$$
e^{-i\langle\xi, \gamma(x, \omega, r)\rangle} \int e^{i[\langle\theta, x-z\rangle+\langle\xi, \gamma(z, \omega, r)\rangle]} p(x, z, \theta) a(z, y, \omega, r, \rho, \xi) d z d \theta .
$$

Notice that if we can choose $B$ in (1.2.a) resp. (1.2.b) arbitrarily close to $-\infty$ then $K_{T}(x, y)$ is $C^{\infty}$, with bounds on the derivatives independent of the size of the supports of our symbols. This is easy to see in the case $\mu<m$. If $\mu=m$, notice that integrating in $d \rho$ is essentially the same as applying a pseudodifferential operator to the bounded family of functions $e^{-i\langle\cdot, \gamma(\cdots, r)\rangle} a(\cdots, r, \cdots)$.

We can assume that our $a(\cdots)=0$ if $|\xi| \leq 1$. Now let $\psi \in C_{0}^{\infty}(\mathbb{R}), \psi=1$ in a neighbourhood of 1 , and with support in $[1 / 2,2]$. We can split the last integral in two pieces, the most interesting being

$$
\int e^{i[\langle\theta, x-z\rangle+\langle\xi, \gamma(z, \omega, r)\rangle]} \psi\left(\frac{|\theta|}{|\xi|}\right) p(x, z, \theta) a(\cdots) d z d \theta,
$$

for $a(\cdots)=a(z, y, \omega, r, \xi)$ resp. $a(z, y, \omega, r, \rho, \xi)$. (The other piece is $\leq c_{N}|\xi|^{-N}$ with each $c_{N}$ depending on finitely many of the constants in (1.2.a) resp. (1.2.b) and can be treated using the previous remark). With a change of variable we obtain

$$
|\xi|^{n} \int e^{i|\xi|[\langle\theta, x-z\rangle+\langle\xi /|\xi|, \gamma(z, \omega, r)\rangle]} \psi(|\theta|) p(x, z,|\xi| \theta) a(\cdot) d z d \theta
$$

with

$$
\begin{aligned}
& \left|\partial_{(z, y, \omega, \theta)}^{\alpha} \partial_{r}^{l} \partial_{\xi}^{\beta}\{\psi(|\theta|) p(x, z,|\xi| \theta) a(z, y, \omega, r, \xi)\}\right| \\
& \quad \leq c_{\alpha, l, \beta} r^{-\mu-l}(1+|\xi|)^{B+\operatorname{ord} P-|\beta|}
\end{aligned}
$$

resp.

$$
\begin{aligned}
& \left|\partial_{(z, y, \omega, \theta, r)}^{\alpha} \partial_{\rho}^{l} \partial_{\xi}^{\beta}\{\psi(|\theta|) p(x, z,|\xi| \theta) a(z, y, \omega, r, \rho, \xi)\}\right| \\
& \quad \leq c_{\alpha, l, \beta}(1+|\rho|)^{-l}(1+|\xi|)^{B+\operatorname{ord} P-|\beta|}
\end{aligned}
$$


with each of the constants in (1.4.a) resp. (1.4.b) depending on finitely many constants in (1.2.a) resp. (1.2.b). The result follows applying the Stationary Phase Theorem (see [S, Corollary 1.1.8, p. 48]). For $Q_{y} K_{T}(x, y)$ the argument is similar.

Splitting any pseudodifferential operator $P=P_{1}+P_{2}$, with $P_{2}$ smoothing and with $P_{1}$ supported near the diagonal, we can reduce Theorem 1 to the following fact:

Proposition 1.2. We consider an operator $T$ with kernel given by (1.1) where $a(x, y, \omega, r, \xi)=0$ if $(x, y, \omega, r)$ does not belong to a small given compact set and (1.2) is satisfied for either $B=\min (k / 2, m-\mu)$ if $m-\mu \neq k / 2$ or $B=k / 2-\delta$ for any $\delta>0$ if $m-\mu=k / 2$. Suppose moreover that each $\gamma_{x}$ satisfies the hypotheses of Theorem 1. Then $T: L^{2}\left(\mathbb{R}^{n}\right) \rightarrow L^{2}\left(\mathbb{R}^{n}\right)$.

The proof of Proposition 1.2 is taken up in section 2 .

\section{Proof of Proposition 1.2}

We can suppose that $a(x, \omega, r, \xi) \neq 0$ only if $\omega$ belongs to a small piece of the sphere $S^{m-1}$ (and if $|\xi|>1$, see the proof of Lemma 1.1). Then we can assume that the tangent spaces $T_{y} \gamma_{x}$, as $x$ varies in a small compact set, are all such that $T_{y} \gamma_{x} \cap V=\{0\}$ with $V$ a given vector space chosen to be perpendicular to one of the $T_{y_{0}} \gamma_{x_{0}}$ and with $T_{y_{0}} \gamma_{x_{0}} \oplus V=T_{y_{0}} S^{n-1}$. We will denote by $C$ an open conical neighbourhood of $V$ in $\mathbb{R}^{n}$. We split $a()=.a_{1}()+.a_{2}($.$) , where a_{1}(x, \omega, r, \xi)=0$ if $\xi \notin C$ and $a_{2}(x, \omega, r, \xi)=0$ if $\xi$ belongs to a thin open cone containing $V$ whose closure is contained in $C$ except for the vertex. In correspondence with this decomposition we obtain two kernels. We begin by discussing the easier, which is

$$
\int e^{i\langle\xi, \gamma(x, \omega, r)-y\rangle} a_{j}(x, y, \omega, r, \xi) r^{m-1} d \xi d \omega d r
$$

when $j=2$; here, for $j=1,2$,

$$
\left|\partial_{(x, y, \omega)}^{\alpha} \partial_{r}^{l} \partial_{\xi}^{\beta} a_{j}(x, y, \omega, r, \xi)\right| \leq c_{\alpha, l, \beta} r^{-\mu-l}(1+|\xi|)^{B-|\beta|} .
$$

The following fact implies that the correponding operator is $L^{2}$ bounded.

\section{Claim 2.1.}

$$
\left|\partial_{(x, y)}^{\alpha_{0}} \partial_{\xi}^{\beta_{0}}\left\{\int e^{i r\left\langle\xi, \gamma^{0}(x, \omega, r)\right\rangle} a_{2}(x, y, \omega, r, \xi) r^{m-1} d \omega d r\right\}\right| \leq c_{\alpha_{0} \beta_{0}}(1+|\xi|)^{-\left|\beta_{0}\right|},
$$

each $c_{\alpha}$ depending on finitely many constants in (2.2).

Proof of the claim. Let $\phi \in C_{0}^{\infty}(\mathbb{R})$ with $\phi=1$ in a neighbourhood of the origin. Performing the above derivatives, we obtain a sum of various terms. Generically we consider the term where, say, $L(x, y)$ derivatives and $L_{1} \xi$ derivatives are applied on the exponential. Then we reduce essentially to evaluating

$$
\begin{aligned}
& I_{1}=\left|\int e^{i r\left\langle\xi, \gamma^{0}(x, \omega, r)\right\rangle} b(x, y, \omega, r, \xi) \phi(|\xi| r) r^{m-1} d \omega d r\right|, \\
& I_{2}=\left|\int e^{i r\left\langle\xi, \gamma^{0}(x, \omega, r)\right\rangle} b(x, y, \omega, r, \xi)(1-\phi(|\xi| r)) r^{m-1} d \omega d r\right|,
\end{aligned}
$$

with $\left|\partial_{(x, y, \omega)}^{\alpha} \partial_{r}^{l} \partial_{\xi}^{\beta} b(x, y, \omega, r, \xi)\right| \leq c_{\alpha, l, \beta} r^{-\mu+L_{1}+L-l}(1+|\xi|)^{B+L+L_{1}-\left|\beta_{0}\right|-|\beta|}$. If we consider the absolute value of the integrand we conclude that $I_{1}$ is essentially 
bounded by $(1+|\xi|)^{B+L+L_{1}-\left|\beta_{0}\right|}(1+|\xi|)^{\mu-m-L_{1}-L}$. We recall that $B \leq m-\mu$. Turning to $I_{2}$, notice that either $\left|\left\langle\xi, \gamma^{0}(x, \omega, r)\right\rangle\right|>c|\xi|$ or $\left|\partial_{\omega}\left\langle\xi, \gamma^{0}(x, \omega, r)\right\rangle\right|>c|\xi|$ for some small $c>0$. Integrating by parts a sufficient number of times with respect to either $d r$ or $d \omega$ and integrating the absolute value as above, we obtain that $I_{2}$ is essentially bounded by $(1+|\xi|)^{\mu-m+B}$.

Notice that, using the previous argument, we obtain

Remark.

$$
\begin{aligned}
& \left|\partial_{(x, y)}^{\alpha_{0}} \partial_{\xi}^{\beta_{0}}\left\{\int e^{i r\left\langle\xi, \gamma^{0}(x, \omega, r)\right\rangle} a(x, y, \omega, r, \xi) \phi(|\xi| r) r^{m-1} d \omega d r\right\}\right| \\
& \quad \leq c_{\alpha_{0} \beta_{0}}(1+|\xi|)^{-\left|\beta_{0}\right|}
\end{aligned}
$$

each $c_{\alpha}$ depending on finitely many constants in (2.2).

We now consider the operator with kernel (2.1) with $j=1$. We consider a $C^{\infty}$ partition of unity $\beta_{0}(t)+\sum_{l \geq 1} \beta_{l}(t)=1$ in $[0, \infty)$ with $\operatorname{supp} \beta_{0} \subset[0,1]$ and $\beta_{l}(t)=\beta\left(2^{-l} t\right)$ with $\operatorname{supp} \beta \subset[1 / 2,2]$, and we define

$$
\left.T_{l} f(x)=\int e^{i\langle\xi, \gamma(x, \omega, r)-y\rangle} a(x, y, \omega, r, \xi) \beta_{l}|\xi|\right) f(y) d \xi d \omega d r d y,
$$

where $a(\cdots)$ stands for $a_{1}(\cdots)$. Proposition 1.2 will be a consequence of the CotlarStein Lemma if we prove the following lemma.

Lemma 2.2. There exists a $c>0$ such that for every $l, j>0$ with $|l-j|>3$ we have:

1. $\left\|T_{l}\right\| \leq c$, where $\|$.$\| is the L^{2}$ norm of the operator;

2. $\left\|T_{l}^{*} T_{j}\right\| \leq c 2^{-\max (j, l)}$;

3. $\left\|T_{l} T_{j}^{*}\right\| \leq c 2^{-\max (j, l)}$.

Proof. We start with (2) and (3). The kernel of $T_{l}^{*} T_{j}$ is

$$
\begin{aligned}
& H(x, y)=\int d \xi d \theta \beta_{l}(|\xi|) \beta_{j}(|\theta|) \int d z d \omega d r d \omega^{\prime} d r^{\prime} r^{m-1}\left(r^{\prime}\right)^{m-1} \\
& \times e^{i\left\{-\left\langle\xi, z-x+r \gamma^{0}(z, \omega, r)\right\rangle+\left\langle\theta, z-y+r^{\prime} \gamma^{0}\left(z, \omega^{\prime}, r^{\prime}\right)\right\rangle\right\}} \bar{a}(z, x, \omega, r, \xi) a\left(z, y, \omega^{\prime}, r^{\prime}, \theta\right) .
\end{aligned}
$$

Let

$$
\begin{aligned}
k\left(x, y, \omega, \omega^{\prime}, r, r^{\prime}, \xi, \theta\right)=\int & e^{i\langle\theta-\xi, z\rangle+r^{\prime}\left\langle\theta, \gamma^{0}\left(z, \omega^{\prime}, r^{\prime}\right)\right\rangle-r\left\langle\xi, \gamma^{0}(z, \omega, r)\right\rangle} \\
& \times \bar{a}(z, x, \omega, r, \xi) a\left(z, y, \omega^{\prime}, r^{\prime}, \xi\right) d z
\end{aligned}
$$

for $\xi, \theta$ with $\beta_{l}(|\xi|) \beta_{j}(|\theta|) \neq 0$. Keeping in mind (2.2) and the fact that, since $|\xi-\theta|$ is large and $r$ and $r^{\prime}$ are small, $\mid \partial_{z}\{$ phase $\}|\approx| \xi-\theta \mid$, it is easy to conclude that the following inequality, which yields (2), holds:

$$
\left|k\left(x, y, \omega, \omega^{\prime}, r, r^{\prime}, \xi, \theta\right)\right| \leq c_{N}(1+|\xi-\theta|)^{-N} r^{-\mu}\left(r^{\prime}\right)^{-\mu} .
$$

The proof of (3) is similar (and easier).

We now turn to the proof of claim (1) of Lemma 2.2. We will consider

$$
\int e^{i\langle\xi, \gamma(x, \omega, r)-y\rangle} a(x, y, \omega, r, \xi) \beta(|\xi| / \tau) r^{m-1} d \xi d \omega d r,
$$


where $a(\cdots)$ satisfies $(2.2)$ and $\tau>1$. If $\phi \in C_{0}^{\infty}(\mathbb{R})$ with $\phi=1$ in a neighbourhood of the origin, let us consider

$$
\int d \xi e^{i\langle\xi, x-y\rangle} \int e^{i r\left\langle\xi, \gamma^{0}(x, \omega, r)\right\rangle} \phi(|\xi| r) a(x, y, \omega, r, \xi) \beta(|\xi| / \tau) r^{m-1} d \omega d r .
$$

If we call the inner integral $B(x, y, \xi)$, the following is true as we remarked after Claim 2.1.

Claim 2.3. $\left|\partial_{(x, y)}^{\alpha} \partial_{\xi}^{\beta} B(x, y, \xi)\right| \leq c_{\alpha}(1+|\xi|)^{-|\beta|}$ uniformly in $\xi, \tau, \epsilon$, where each $c_{\alpha}$ depends on finitely many of the constants in (2.2).

For the proof see the first part of the proof of Claim 2.1

Finally we turn to

$$
\int e^{i\langle\xi, \gamma(x, \omega, r)-y\rangle}(1-\phi(|\xi| r)) a(x, y, \omega, r, \xi) \beta(|\xi| / \tau) r^{m-1} d \xi d \omega d r .
$$

Recall that $a(x, \omega, r, \xi)=0$ if $|\xi| \leq 1$ and if $\xi$ is outside a closed cone in $\mathbb{R}^{n}-0$ having $C$ (the cone introduced at the beginning of section 2 ) as a neighbourhood. We decompose $a(\cdot)=\sum a_{j}(\cdot)$ with each $a_{j}(\cdot)$ having $\xi$-support contained in a sufficiently thin cone. Our kernel (2.4) is then a sum of

$$
\begin{aligned}
& \int e^{i\langle\xi, x-y\rangle}(1-\phi(|\xi| r)) \beta(|\xi| / \tau) I_{j}(x, y, \xi, r, \tau) r^{m-1} d \xi d r \\
& \text { with } \quad I_{j}(x, y, \xi, r, \tau)=\int e^{i r\left\langle\xi, \gamma^{0}(x, \omega, r)\right\rangle} a_{j}(x, y, \omega, r, \xi) d \omega .
\end{aligned}
$$

For any $I_{j}$ one of the following two cases I or II holds. In case $\mathrm{I}\left|\partial_{\omega}\left\langle\xi, \gamma^{0}(x, \omega, r)\right\rangle\right| \geq$ $c>0$ in the support of $a_{j}(\cdots)$, and we can show that

$$
\left|\partial_{\xi}^{\alpha} I_{j}(x, y, \xi, r, \tau)\right| \leq c_{\alpha N} \tau^{-N+B} r^{-N-\mu}
$$

(see [S, Proposition 4, p. 341]). In case II we can suppose that for some choice of coordinates $\omega=\left(\omega^{\prime}, \omega^{\prime \prime}\right) \in \mathbb{R}^{k} \times \mathbb{R}^{m-k-1}$ in the support of $a_{j}(\cdots)$ we have $\operatorname{rk} \partial_{\omega^{\prime} \omega^{\prime}}^{2}\left\langle\xi, \gamma^{0}().\right\rangle=k$, and the set of points satisfying $\omega^{\prime}=\omega^{\prime}\left(x, \omega^{\prime \prime}, r, \xi\right)$, for some function $\omega(\cdot)$, is exactly the set where $\partial_{\omega^{\prime}}\left\langle\xi, \gamma^{0}\left(x, \omega^{\prime}, \omega^{\prime \prime}\right)\right\rangle=0$. Still in case II we write

$$
I_{j}(x, y, \xi, r, \tau)=\int d \omega^{\prime \prime} I_{j}\left(x, y, \xi, r, \tau, \omega^{\prime \prime}\right)
$$

where

$$
I_{j}\left(x, y, \xi, r, \tau, \omega^{\prime \prime}\right)=\int e^{i r\left\langle\xi, \gamma^{0}(x, \omega, r)\right\rangle} a_{j}(x, y, \omega, r, \xi) d \omega^{\prime} .
$$

Then, essentially by the stationary phase theorem and by estimates (2.2) we obtain

$$
\left|\partial_{\xi}^{\alpha}\left\{e^{-i r\left\langle\xi, \gamma^{0}\left(x, \omega^{\prime}\left(x, \omega^{\prime \prime}, r, \xi\right), \omega^{\prime \prime}, r\right)\right\rangle} I_{j}\left(x, y, \xi, r, \tau, \omega^{\prime \prime}\right)\right\}\right| \leq c_{\alpha} \tau^{-k / 2+B-|\alpha|} r^{-k / 2-\mu} ;
$$

see [S, Corollary 1.18, p. 48]. To estimate the $L^{2}$ norm of the operator with kernel (2.5) we can use Young's inequality. We consider case II, which is the more difficult. We write

$$
\begin{aligned}
& \int e^{i\left\langle\xi, x-y+r \gamma^{0}\left(x, \omega^{\prime}\left(x, \omega^{\prime \prime}, r, \xi\right), \omega^{\prime \prime}, r\right)\right\rangle}(1-\phi(|\xi| r)) \beta(|\xi| / \tau) \\
& \quad \times\left\{e^{-i r\left\langle\xi, \gamma^{0}\left(x, \omega^{\prime}\left(x, \omega^{\prime \prime}, r, \xi\right), \omega^{\prime \prime}, r\right)\right\rangle} I_{j}\left(x, y, \xi, r, \tau, \omega^{\prime \prime}\right)\right\} r^{m-1} d \xi d \omega^{\prime \prime} d r .
\end{aligned}
$$


This is bounded by (at this point we use the fact that for $\omega^{\prime}=\omega^{\prime}\left(x, \omega^{\prime \prime}, r, \xi\right)$, for some function $\omega(\cdot)$, is exactly the set where $\left.\partial_{\omega^{\prime}}\left\langle\xi, \gamma^{0}\left(x, \omega^{\prime}, \omega^{\prime \prime}\right)\right\rangle=0\right)$

$$
\begin{aligned}
C_{N} \int_{r \geq \tau^{-1},|\xi| \approx \tau}(1+\tau \mid x-y & \left.+r \gamma^{0}\left(x, \omega^{\prime}\left(x, \omega^{\prime \prime}, r, \xi\right), \omega^{\prime \prime}, r\right) \mid\right)^{-N} \\
& \times \tau^{-k / 2+B} r^{-k / 2-\mu} r^{m-1} d \xi d \omega^{\prime \prime} d r .
\end{aligned}
$$

If we integrate this quantity also with respect to either $d x$ or $d y$, we obtain a number uniformly bounded by a constant, independent of $y$ or $x$ (the case of the $d y$ integration is immediate, and in the case of the $d x$ integration one uses the fact that $r \ll 1)$.

\section{Proof of Theorem 2}

By Lemma 1.1 we are reduced to considering the $L^{p}$ boundedness of operators with kernel (1.1.b) with (1.2.b) satisfied for $B=0$. Consider now $\phi \in C_{0}^{\infty}(\mathbb{R})$ with $\phi=1$ in a neighbourhood of the origin. We split the kernel into two pieces, (3.1) and (3.2) below. We begin considering

$$
\int e^{i\left\langle\xi, x-y+r \gamma^{0}(x, \omega, r)\right\rangle} \phi(|\xi| r) a(x, y, \omega, r, \rho, \xi) d \xi d \omega d r d \rho
$$

and from $e^{i\left\langle\xi, r \gamma^{0}(x, \omega, r)\right\rangle}=1+O(|\xi| r)$ we split (3.1) into two pieces

$$
I_{j}=\int e^{i\langle\xi, x-y\rangle} B_{j}(x, y, \xi) d \xi
$$

for $j=1,2$, with

$$
\begin{aligned}
& B_{1}(x, y, \xi)=\int e^{i \rho r} \phi(|\xi| r) a(x, y, \omega, r, \rho, \xi) d \omega d r d \rho \\
& B_{2}(x, y, \xi)=\int e^{i \rho r} O(|\xi| r) \phi(|\xi| r) a(x, y, \omega, r, \rho, \xi) d \omega d r d \rho .
\end{aligned}
$$

That the operator with kernel (3.1) is $L^{p}$ bounded for every $1<p<\infty$ follows from the following :

Claim. $\left|\partial_{(x, y)}^{\alpha} \partial_{\xi}^{\beta} B_{j}(x, y, \xi)\right| \leq c_{\alpha \beta}(1+|\xi|)^{-|\beta|}$ for $j=1,2$.

Proof. Consider

$$
\partial_{(x, y)}^{\alpha} \partial_{\xi}^{\beta} B_{1}(x, y, \xi)=\int e^{i \rho r} \partial_{(x, y)}^{\alpha} \partial_{\xi}^{\beta}\{\phi(|\xi| r) a(x, y, \omega, r, \rho, \xi)\} d \omega d r d \rho .
$$

For simplicity suppose $a(x, y, \omega, r, \rho, \xi)=\phi(r) a(x, y, \omega, \rho, \xi)$, and let us omit the factor $\phi(r)$ since $|\xi| \gg 1$. Let $\hat{a}(x, y, \omega, r, \xi)=\int e^{i \rho r} \hat{a}(x, y, \omega, \rho, \xi) d \rho$. The desired estimates for $j=1$ follow from the fact that

$$
\left|\int_{\epsilon<|r|<\eta} \partial_{(x, y)}^{\alpha} \partial_{\xi}^{\beta} \hat{a}(x, y, \omega, r, \xi) d r\right| \leq c_{\alpha \beta}(1+|\xi|)^{-|\beta|}
$$

see p. 291, [St], Proposition 2.

Just by taking the absolute value of the integrand in

$$
\partial_{(x, y)}^{\alpha} \partial_{\xi}^{\beta} B_{2}(x, y, \xi)=\int_{|r| \leq 1 /|\xi|} \int e^{i \rho r} \partial_{(x, y)}^{\alpha} \partial_{\xi}^{\beta}\{O(|\xi| r) \phi(|\xi| r) \hat{a}(x, y, \omega, r, \xi)\} d \omega d r
$$


since $O(|\xi| r) \phi(|\xi| r) a(x, y, \omega, r, \xi)$ can be thought as homogeneous of degree 0 in $r$, we obtain that it is bounded by $(1+|\xi|)^{-|\beta|}$.

We turn now to the kernel

$$
\int e^{i\left\langle\xi, x-y+r \gamma^{0}(x, \omega, r)\right\rangle+\rho r}(1-\phi(|\xi| r)) a(x, y, \omega, r, \rho, \xi) d \xi d \omega d r d \rho .
$$

We embed (3.2) in an analytic family of kernels, replacing $a($.$) in (3.2) by$

$$
a_{z}(x, y, \omega, r, \rho, \xi)=a(x, y, \omega, r, \rho, \xi)\left(1+|\xi|^{2}\right)^{-z / 2}\left(1+|\rho|^{2}\right)^{\frac{z}{2}} .
$$

If now

$$
\hat{a}_{z}(x, y, \omega, r, \xi)=\int e^{i \rho r} \hat{a}_{z}(x, y, \omega, r, \rho, \xi) d \rho,
$$

we have

$$
\left|\partial_{(x, y, \omega)}^{\alpha} \partial_{r}^{l} \partial_{\xi}^{\beta} \hat{a}_{z}(x, y, \omega, r, \xi)\right| \leq c_{\alpha l \beta}(z) r^{-1-\operatorname{Re} z-l}(1+|\xi|)^{-\operatorname{Re} z-|\beta|},
$$

where the $c_{\alpha l \beta}(z)$ as well as all the constants dependent on the parameter $z$ introduced below grow polynomially as $|z| \rightarrow \infty$. Writing

$$
B_{z}(x, y, \xi)=\int e^{i r\left\langle\xi, \gamma^{0}(x, \omega, r)\right\rangle}(1-\phi(|\xi| r)) \hat{a}_{z}(x, y, \omega, r, \xi) d \omega d r,
$$

our kernels are of the form $\int e^{i\langle\xi, x-y\rangle} B_{z}(x, y, \xi) d \xi$. Let us denote by $\left\{T_{z}\right\}$ the corresponding family of operators. An elementary calculation shows that

$$
\left|\partial_{(x, y)}^{\alpha} \partial_{\xi}^{\beta} B_{z}(x, y, \xi)\right| \leq c_{\alpha \beta}(z)(1+|\xi|)^{-|\beta|} \text { for } \operatorname{Re} z>|\alpha|+|\beta| .
$$

Moreover, with an argument as in Claim 2.1, these inequalities hold for any $\operatorname{Re} z>0$ if $\xi$ is not, say, in the cone $C$ introduced at the beginning of section 2 .

Using the proof of Theorem 1 and complex interpolation, we conclude that for any $z$ with $-\epsilon \leq \operatorname{Re} z \leq \epsilon$ for some $\epsilon>0,\left\|T_{z}\right\|_{L^{2}} \leq c(z)$.

What remains to be proved are the $L^{p}$ estimates when $a(x, y, \omega, r, \rho, \xi)=0$ for $\xi \notin C$ (also for $\omega$ outside a small portion of $S^{m-1}$ ). In this part of the proof we will reduce essentially to the arguments on pp. 140-144 in [PS]. First of all there is a choice of coordinates, where if $\gamma^{0}(x, \omega, r)=\left(\gamma_{1}^{0}(x, \omega, r), \gamma_{2}^{0}(x, \omega, r)\right) \in \mathbb{R}^{n-m} \times \mathbb{R}^{m}$, then $\left|\gamma_{1}^{0}(\cdot)\right| \ll 1$ and $\left|\gamma_{2}^{0}(\cdot)\right| \approx 1$, and for any fixed $\mathrm{x}$ the map $(r, \omega) \rightarrow r \gamma_{2}^{0}(x, \omega, r)$ represents basically the spherical coordinates. Notice that $\Gamma$ is given parametrically by $y=x+r \gamma^{0}(x, \omega, r)$; we can express $\omega=\omega\left(x, x_{2}-y_{2}\right)$ and $r=r\left(x, x_{2}-y_{2}\right)$ with $\left|\partial_{(x, y)}^{\alpha} r(\cdot)\right| \leq c_{\alpha}\left|x_{2}-y_{2}\right|^{-|\alpha|+1}$ and $\left|\partial_{(x, y)}^{\alpha} \omega(\cdot)\right| \leq c_{\alpha}\left|x_{2}-y_{2}\right|^{-|\alpha|}$; and after a substitution we can assume $\Gamma$ is defined by the equation $x_{1}-y_{1}+S\left(x, x_{2}-y_{2}\right)=0$, where $\left(x_{1}, x_{2}\right) \in \mathbb{R}^{n-m} \times \mathbb{R}^{m}$, and $S\left(x, x_{2}-y_{2}\right)$ is Lipschitz and has absolute value much smaller than $\left|x_{2}-y_{2}\right|$.

We now define the map $d(x, y)=\left|x_{1}-y_{1}+S\left(x, x_{2}-y_{2}\right)\right|+\left|x_{2}-y_{2}\right|$. It is easy to see that $d(\cdot)$ satifies the following three properties: for an appropriate constant $c$

1. $d(x, y)=0 \quad \Longrightarrow \quad x=y$;

2. $d(x, y) \leq c d(y, x)$;

3. $d(x, y) \leq c(d(x, w)+d(w, y))$.

Therefore $d(\cdot)$ is what is called a quasidistance - see [St, p. 10]. 
Lemma 3.1. Fix $\operatorname{Re} z>0$. Let $\alpha$ be a multiindex with $|\alpha| \leq 1$. The following inequalities hold: if $\left|x_{2}-y_{2}\right| \geq\left|x_{1}-y_{1}+S\left(x, x_{2}-y_{2}\right)\right|$, then

$$
\left|\partial_{(x, y)}^{\alpha} K_{z}(x, y)\right| \leq c_{\alpha}(z)\left|x_{2}-y_{2}\right|^{-m-\operatorname{Re} z}\left|x_{1}-y_{1}+S\left(x, x_{2}-y_{2}\right)\right|^{-(n-m)+\operatorname{Re} z-|\alpha|},
$$

and if $\left|x_{2}-y_{2}\right| \leq\left|x_{1}-y_{1}+S\left(x, x_{2}-y_{2}\right)\right|$, then

$$
\left|\partial_{(x, y)}^{\alpha} K_{z}(x, y)\right| \leq c_{\alpha}(z)\left|x_{1}-y_{1}+S\left(x, x_{2}-y_{2}\right)\right|^{-n-|\alpha|} .
$$

Proof. We start with the case $\left|x_{2}-y_{2}\right| \leq\left|x_{1}-y_{1}+S\left(x, x_{2}-y_{2}\right)\right|$. Then

$$
\left|x_{1}-y_{1}+S\left(x, x_{2}-y_{2}\right)\right| \approx\left|x_{1}-y_{1}\right| \text {. }
$$

Given

$$
K_{z}(x, y)=\int e^{\left\langle\xi, x-y+r \gamma^{0}(x, \omega, r)\right\rangle}(1-\phi(|\xi| r)) \hat{a}_{z}(x, y, \omega, r, \xi) d \omega d r d \xi,
$$

the estimate for the case $|\alpha|=0$ is obtained in a standard way as follows. We integrate $d \xi$ separately in $|\xi| \leq\left|x-y+r \gamma^{0}(x, \omega, r)\right|^{-1} \approx\left|x_{1}-y_{1}\right|^{-1}$ and in the complementary region, using an appropriate smooth partition of unity. In the first case, integrating first $d r d \omega$ and then $d \xi$, we find the integral is bounded, up to a constant factor, by $\left|x_{1}-y_{1}\right|^{-n}$. Turning to the second case, we first integrate by parts $d \xi$ and then we integrate $d r d \omega$, obtaining something of the form $\int\left|x_{1}-y_{1}\right|^{-N}|\xi|^{-N} d \xi$, where essentially $|\xi| \geq\left|x_{1}-y_{1}\right|^{-1}$, and so we find the desired bound. When $|\alpha|=1$ the argument is essentially the same.

We turn now to the case $\left|x_{2}-y_{2}\right| \geq\left|x_{1}-y_{1}+S\left(x, x_{2}-y_{2}\right)\right|$. As above we discuss the case $|\alpha|=0$ (the case $|\alpha|=1$ admits a similar proof). As mentioned above, we can assume that $\xi=\left(\xi_{1}, \xi_{2}\right) \in \mathbb{R}^{n-m} \times \mathbb{R}^{m}$ with $c\left|\xi_{1}\right| \geq\left|\xi_{2}\right|$. We write $w_{2}=-r \gamma^{0}(x, \omega, r)$, and after a change of coordinates we can assume (3.3) is essentially

$$
\int e^{\left\langle\xi_{1}, x_{1}-y_{1}+S\left(x, w_{2}\right)\right\rangle} e^{\left\langle\xi_{2}, x_{2}-y_{2}-w_{2}\right\rangle}\left(1-\phi\left(\left|\xi_{1}\right|\left|w_{2}\right|\right)\right) b_{z}\left(x, y, w_{2}, \xi\right) d \xi_{1} d \xi_{2} d w_{2}
$$

with

$$
\left|\partial_{(x, y)}^{\alpha} \partial_{w_{2}}^{\beta} \partial_{\xi}^{\gamma} b_{z}\left(x, y, w_{2}, \xi\right)\right| \leq c_{\alpha \beta \gamma}(z)\left|w_{2}\right|^{-m-\operatorname{Re} z-|\beta|}(1+|\xi|)^{-\operatorname{Re} z-|\gamma|} .
$$

We integrate $d \xi_{2} d w_{2}$, obtaining $\int e^{\left\langle\xi_{1}, x_{1}-y_{1}+S\left(x, x_{2}-y_{2}\right)\right\rangle} A_{z}\left(x, y, \xi_{1}\right) d \xi_{1}$ with

$$
\begin{aligned}
& A_{z}\left(x, y, \xi_{1}\right)=\int e^{\left\langle\xi_{1}, S\left(x, w_{2}\right)-S\left(x, x_{2}-y_{2}\right)\right\rangle} \\
& \quad \times\left(1-\phi\left(\left|\xi_{1}\right|\left|w_{2}\right|\right)\right)\left|w_{2}\right|^{-m-\operatorname{Re} z}\left|\xi_{1}\right|^{m} \tilde{b}_{z}\left(x, y,\left|\xi_{1}\right|\left(x_{2}-y_{2}-w_{2}\right), \xi_{1}\right) d w_{2},
\end{aligned}
$$

where here

$$
\left|\partial_{\xi_{1}}^{\beta} \tilde{b}_{z}\left(x, y, v_{2}, \xi_{1}\right)\right| \leq c_{\beta N}(z)\left(1+\left|v_{2}\right|\right)^{-N}\left(1+\left|\xi_{1}\right|\right)^{-\operatorname{Re} z-|\beta|} .
$$

We claim that

$$
\left|\partial_{\xi_{1}}^{\beta} A_{z}\left(x, y, \xi_{1}\right)\right| \leq c_{\beta}(z)\left|x_{2}-y_{2}\right|^{-m-\operatorname{Re} z}\left(1+\left|\xi_{1}\right|\right)^{-\operatorname{Re} z-|\beta|} .
$$

We start by considering the case $|\beta|=0$. Then, taking absolute values in (3.4), we find $\left|A_{z}\left(x, y, \xi_{1}\right)\right|$ is bounded up to a constant factor by

$$
\left|\xi_{1}\right|^{m+\operatorname{Re} z} \int\left(1-\phi\left(\left|w_{2}\right|\right)\right)\left|w_{2}\right|^{-m-\operatorname{Re} z}\left|\tilde{b}_{z}\left(x, y, u_{2}-w_{2}, \xi\right)\right| d w_{2}
$$


with $u_{2}=\left|\xi_{1}\right|\left(x_{2}-y_{2}\right)$. Because of (3.5), (3.6) is bounded by $\left|u_{2}\right|^{-m-\operatorname{Re} z}\left|\xi_{1}\right|^{m}$, and so we find the desired bound. For $\beta \neq 0$ we reduce to bound

$$
\begin{aligned}
& \int\left|w_{2}\right|^{-m-\operatorname{Re} z}\left|\xi_{1}\right|^{-|\beta|-\operatorname{Re} z+m} \\
& \quad \times\left(\left|\xi_{1}\right|^{\left|\beta_{1}\right|+\left|\beta_{2}\right|}\left|x_{2}-y_{2}-w_{2}\right|^{\left|\beta_{1}\right|+\left|\beta_{2}\right|}\left(1+\left|\xi_{1}\right|\left|x_{2}-y_{2}-w_{2}\right|\right)^{-N}\right) d w_{2}
\end{aligned}
$$

with $\left|\beta_{1}\right|$ the number of derivatives applied on the exponential and $\left|\beta_{2}\right|$ the number of derivatives applied on $\tilde{b}_{z}\left(\cdots\left|\xi_{1}\right|\left(x_{2}-y_{2} w_{2}\right), \cdot\right)$. The estimate is then similar to the previous one.

Finally we prove

Lemma 3.2. Fix $\operatorname{Re} z>0$. Then

$$
\int\left|K_{z}(x, y)-K_{z}\left(x, y^{\prime}\right) d x\right| \leq c(z)
$$

and

$$
\int\left|K_{z}(y, x)-K_{z}\left(y^{\prime}, x\right) d x\right| \leq c(z),
$$

where the integrals are over the region $d(x, y)>\tilde{c} d\left(y, y^{\prime}\right)$ for any given $y$ and $y^{\prime}$, and where $\tilde{c}$ is an appropriate constant.

Proof. Here we prove only the first of the two inequalities. In different formulas $c$ will indicate different constants. The proof is essentially the same as [PS, Lemma 4, p. 141]. We consider $\psi(t)$ in $C^{\infty}(\mathbb{R})$ with $\psi=1$ far from 0 , and $\psi=0$ near 0 , and $\psi_{j}(t)=\psi\left(2^{j+1} t\right)-\psi\left(2^{j} t\right)$. We consider then, for $\sigma=\frac{\left|x_{1}-y_{1}+S\left(x, x_{2}-y_{2}\right)\right|}{\left|x_{2}-y_{2}\right|}$, the kernels $K_{z}(x, y) \psi(\sigma)$ and $K_{z}^{j}(x, y) \psi_{j}(\sigma)$. We can now prove appropriate inequalities separately for these kernels. For the first one, by Lemma 2.3.1, we can use the theory in Ch. 1 of [St]. Each $K_{z}^{j}$ can be treated as follows. Split the region of integration into two pieces. One piece, denoted here by $\mathrm{I}$, is defined by $\underline{c} 2^{-j} d(x, y) \leq d\left(y, y^{\prime}\right)$, with $\underline{c}$ a sufficiently small positive constant, while the complementary regin will be denoted here by II.

Considering I, we bound $\int_{\mathrm{I}}\left|K_{z}^{j}(x, y)\right| d x+\int_{\mathrm{I}}\left|K_{z}^{j}\left(x, y^{\prime}\right)\right| d x$. Now

$$
\int_{\mathrm{I}}\left|K_{z}^{j}(x, y)\right| d x \leq c \int\left|x_{2}-y_{2}\right|^{-m-\operatorname{Re} z}\left|x_{1}-y_{1}+S\left(x, x_{2}-y_{2}\right)\right|^{-(n-m)+\operatorname{Re} z} d x .
$$

Since I can be described in the form $c_{1} d\left(y, y^{\prime}\right) \leq\left|x_{2}-y_{2}\right| \leq 2^{j} d\left(y, y^{\prime}\right)$ and $\left|x_{2}-y_{2}\right|$ $\approx 2^{j}\left|x_{1}-y_{1}+S\left(x, x_{2}-y_{2}\right)\right|$, through an elementary calculation we find the bound $j 2^{-j \operatorname{Re} z}$. Turning to $\int_{\mathrm{I}}\left|K_{z}^{j}\left(x, y^{\prime}\right)\right| d x$, the bound and the argument are the same because region I is contained in a region defined by $d\left(y, y^{\prime}\right)<d\left(x, y^{\prime}\right)<c 2^{j} d\left(y, y^{\prime}\right)$ (this uses the fact that $\tilde{c}$ is large).

We turn now to region II. In it $\frac{\left|x_{1}-y_{1}+S\left(x, x_{2}-y_{2}\right)\right|}{\left|x_{2}-y_{2}\right|} \approx 2^{-j}$, and we claim that for an appropriate choice of the constants in the proof the same is true on replacing $y$ by $y^{\prime}$ or by any point in the straight arc joining them in $\mathbb{R}^{n}$. This can be understood as follows. First of all, observe that inequalities of the form

$$
\left|y_{2}^{\prime}-y_{2}\right| \leq d\left(y, y^{\prime}\right)<\underline{c} 2^{-j} d(x, y) \leq c \underline{c} 2^{-j}\left|x_{2}-y_{2}\right|
$$


imply, for $\underline{c} 2^{-j}$ sufficiently small, $\left|x_{2}-y_{2}\right| \approx\left|x_{2}-y_{2}^{\prime}\right|$. On the other hand,

$$
\left|y_{1}-S\left(x, x_{2}-y_{2}\right)-y_{1}^{\prime}+S\left(x, x_{2}-y_{2}^{\prime}\right)\right| \leq c\left|y-y^{\prime}\right| \approx c d\left(y^{\prime}, y\right) .
$$

Now

$$
d\left(y^{\prime}, y\right)<\underline{c} 2^{-j} d(x, y) \leq c \underline{c} 2^{-j}\left|x_{2}-y_{2}\right|
$$

and since $\left|y_{1}-S\left(x, x_{2}-y_{2}\right)\right| \approx 2^{-j}\left|x_{2}-y_{2}\right|$, for $\underline{c}$ sufficiently small

$$
\left|y_{1}^{\prime}-S\left(x, x_{2}-y_{2}^{\prime}\right)\right| \approx 2^{-j}\left|x_{2}-y_{2}^{\prime}\right| .
$$

Thus we have proved the above claim. Now

$$
\begin{aligned}
&\left|K_{z}^{j}(x, y)-K_{z}^{j}\left(x, y^{\prime}\right)\right| \leq c\left|x_{1}-y_{1}+S\left(x, x_{2}-y_{2}\right)\right|^{-n-1} 2^{(n-m-\operatorname{Re} z) j} d\left(y^{\prime}, y\right), \\
&\left|K_{z}^{j}(x, y)-K_{z}^{j}\left(x, y^{\prime}\right)\right| \leq c\left|x_{1}-y_{1}+S\left(x, x_{2}-y_{2}^{\prime}\right)\right|^{-n} 2^{(n-m+1-\operatorname{Re} z) j},
\end{aligned}
$$

and so

$$
\left|K_{z}^{j}(x, y)-K_{z}^{j}\left(x, y^{\prime}\right)\right| \leq c\left|x_{1}-y_{1}+S\left(x, x_{2}-y_{2}\right)\right|^{-n-\epsilon} 2^{(n-m+\epsilon-\operatorname{Re} z) j}\left(d\left(y^{\prime}, y\right)\right)^{\epsilon} .
$$

Integrating this last quantity in $d x$ in the region $\left|x_{1}-y_{1}+S\left(x, x_{2}-y_{2}\right)\right| \approx 2^{-j}\left|x_{2}-y_{2}\right|$ and $\left|x_{2}-y_{2}\right|>c d\left(y^{\prime}, y\right)$, we obtain essentially the bound $2^{(\epsilon-\operatorname{Re} z) j}$. Picking $\epsilon<\operatorname{Re} z$ and adding over all the $j$ 's gives the desired result

From the above discussion and from the theory of Calderón-Zygmund kernels we conclude that $\left\|T_{z}\right\|_{L^{p} \rightarrow L^{p}} \leq c(z, p)$ when $0<\operatorname{Re} z \leq \epsilon$ (here the constant depends polynomially on $z$ ). Theorem 2 follows by complex interpolation.

\section{Proof of Theorem 3}

Theorem 3 now follows by interpolation using the previous theorems. By the previous proofs it is enough to consider the case when

$$
K_{T}(x, y)=\int e^{i\langle\xi, \gamma(x, \omega, r)-y\rangle}\left(1-\phi(r|\xi|) a(x, y, \omega, r, \xi) r^{m-1} d \xi d \omega d r,\right.
$$

where $a(x, y, \omega, r, \xi)=0$ if $(x, y, \omega, r)$ does not belong to some small compact set and

$$
\left|\partial_{(x, y, \omega)}^{\alpha} \partial_{r}^{l} \partial_{\xi}^{\beta} a(x, y, \omega, r, \xi)\right| \leq c_{\alpha, l, \beta} r^{-\mu-l}(1+|\xi|)^{-|\beta|} .
$$

Consider now a family of operators $\left\{T_{z}\right\}$ whose kernels are obtained by replacing $a($.$) with a_{z}(x, y, \omega, r, \xi)=\exp \left(z^{2}\right) a(x, y, \omega, r, \xi)\left(1+|\xi|^{2}\right)^{-z / 2} r^{-z}$. By the proofs of the previous theorems we have $\left\|(1-\Delta)^{(z+m-\mu) / 2} T_{z}\right\|_{L_{r}^{2}, L_{r}^{2}} \leq c$ for Re $z$ any negative number larger than $m-\mu-k / 2$. Moreover, $\left\|T_{z}\right\|_{L_{r}^{p}, L_{r}^{p}} \leq c_{p}$ for $\operatorname{Re} z<m-\mu$ (for any such $z$ we can apply the theory developed in the proof of Theorem 2). The result follows by interpolating between these extremes.

\section{REFERENCES}

[G] V.Guillemin, Cosmology in (2+1)-Dimensions, Cyclic Models, and Deformations, Princeton Univ. Press, 1989. MR 91k:58140

[GU1] A.Greenleaf and G.Uhlmann, Estimates for singular Radon transforms and pseudodifferential operators with singular symbols, J.Funct.Anal. 89 (1990), 202-232. MR 91i:58146

[GU2] A.Greenleaf and G.Uhlmann, Composition of some singular Fourier integral operators and estimates for restricted x-ray transforms, II, Duke Math.Jour. 64 (1991), 415-444. MR 93b:58146

[PS] D.H.Phong and E.M.Stein, Hilbert integrals, singular integrals, and Radon transforms I, Acta Math. 157 (1986), 99-157. MR 88i:42028a 
[S] C.D.Sogge, Fourier Integrals in Classical Analysis, Cambridge Univ. Press, 1993. MR 94c: 35178

[SS] C.D.Sogge and E.M.Stein, Averages over hypersurfaces: Smoothness of generalized Radon transforms, J. Analyse Math. 54 (1990), 165-188. MR 91i:58145

[St] E.M.Stein, Harmonic Analysis: real variable methods, orthogonality, and oscillatory integrals, Princeton Univ. Press, 1993. MR 95c:42002

Department of Mathematics, Columbia University, New York, New York 10027

Current address: Department of Mathematics, Princeton University, Princeton, New Jersey 08544

E-mail address: cuccagna@math.princeton.edu 